\title{
Práticas de ensino-aprendizagem com base em cenários reais*
}

Suzely Adas Saliba Moimaz ${ }^{1}$

Nemre Adas Saliba²

Lívia Guimarães Zina ${ }^{3}$

Orlando Saliba ${ }^{4}$

Cléa Adas Saliba Garbin ${ }^{5}$

MOIMAZ, S.A.S. et al. Teaching-learning practices based on real scenarios. Interface Comunic., Saude, Educ., v.14, n.32, p.69-79, jan./mar. 2010.

The teaching-research-extension process carried out with immersion of subjects in real scenarios enables university-community integration, thereby increasing social insertion. The aim of this study was to present the experience from a project conducted in five Brazilian municipalities, with participation of teachers and postgraduate and undergraduate students, in partnership with municipal authorities and healthcare workers as a scenario for teaching-research within the Brazilian health system. Workshops and training courses were conducted among healthcare teams, managers and health counselors, and technical visits were made to the municipalities for situational assessments, activity supervision, user satisfaction evaluations and formation of popular leaderships. Improvements in service organization and stimulation of strengthened links between users and service providers were observed. This experience served as a learning and research laboratory: science was conducted from in situ experience of health system realities, thereby contributing towards better humanitarian professional training based on real scenarios.

Keywords: National Health System. Teaching. Academies and institutes. Health manpower. Health service research.
O processo ensino-pesquisa-extensão realizado com a imersão dos sujeitos em cenários reais possibilita a integração universidade-comunidade, ampliando a inserção social. O objetivo deste trabalho foi apresentar a experiência de um projeto conduzido em cinco municípios brasileiros, com a participação de docentes, pósgraduandos e acadêmicos, em parceria com prefeituras municipais e trabalhadores de saúde como cenário de ensino-pesquisa no SUS. Foram realizadas oficinas e cursos de capacitação com as equipes de saúde, gestores e conselheiros de saúde, visitas técnicas aos municípios para avaliação situacional e supervisão das atividades, avaliação da satisfação dos usuários e formação de lideranças populares. Observou-se a melhoria na organização dos serviços e estímulo ao fortalecimento do vínculo entre os usuários e prestadores de serviços. Essa experiência serviu como laboratório de aprendizagem e pesquisa, fazendo-se ciência a partir da vivência in loco da realidade do SUS e contribuindo para uma formação professional mais humanitária baseada em cenários reais.

Palavras-chave: Sistema Único de Saúde. Ensino. Academias e Institutos. Recursos humanos em saúde. Pesquisa sobre serviços de saúde.

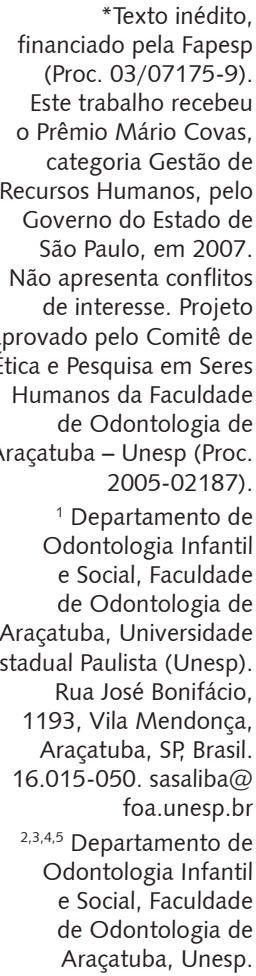

v.14, n.32, p.69-79, jan./mar. 2010 


\section{Introdução}

O modelo de atenção à saúde centrado na doença não conseguiu responder às necessidades de saúde das populações. O movimento de reforma sanitária lutou para que não fossem mais importados modelos de atenção à saúde, e sugeriu uma ampla reforma na estrutura deste setor (González, 2001). No Brasil, a partir da versão mais recente da Constituição Federal de 1988, ficou determinada a criação de um novo sistema de saúde, o Sistema Único de Saúde (SUS), tendo como base a descentralização e fortalecimento do poder municipal, além do estabelecimento da participação da sociedade organizada na administração do setor saúde e o controle social, visando a formulação, implantação, controle e avaliação das políticas de saúde (Escorel et al., 2007). Em 1990, foram publicadas as leis 8080/90 (Brasil, 1990a) e 8142/90 (Brasil, 1990b) que institucionalizaram e regulamentaram a participação popular e o controle social na gestão da saúde, tendo como instâncias legalmente instituídas as Conferências e os Conselhos de Saúde. Estes são descritos como órgãos permanentes, deliberativos e normativos do Sistema Único de Saúde no âmbito municipal, que tem por competência formular estratégias e controlar a execução da política de saúde do município, inclusive nos seus aspectos econômicos e financeiros (Brasil, 2006). As diretrizes do novo sistema de saúde serviram de base para estruturar as mudanças necessárias no setor saúde, favorecendo a descentralização das ações, de forma que cada município passasse a gerir as ações em saúde conforme as necessidades locais.

Visando a consolidação do sistema de saúde, o Ministério da Saúde propôs aos municípios, em 1994, o Programa Saúde da Família (PSF) - posteriormente denominado como Estratégia Saúde da Família (ESF) - com o objetivo de reorganizar a prática de cuidado à saúde em novas bases (Escorel et al., 2007). Uma das demandas da ESF é o retorno da inclusão da família como partícipe do processo saúde-doença e em cujo espaço busca-se o desenvolvimento de ações preventivas, curativas e de reabilitação (Oliveira, Spiri, 2006). Segundo as diretrizes dessa estratégia, a equipe de saúde da família deve estar preparada: para o conhecimento da realidade das famílias pelas quais é responsável; para a identificação dos problemas de saúde mais prevalentes e das situações de risco às quais a população está exposta; para a elaboração de um plano, com a participação da comunidade, que se destine ao enfrentamento dos determinantes do processo saúde-doença; para a prestação de assistência integral e para o desenvolvimento de ações educativas. A saúde bucal foi introduzida na ESF a partir do ano 2000, sendo que, em muitos municípios brasileiros, ainda está em fase de implantação (Souza, Roncalli, 2007).

Nesse mesmo momento, o Ministério da Educação deliberou sobre as Novas Diretrizes Curriculares Nacionais dos cursos de graduação da área de saúde, nas quais ficou evidente a preocupação com o estabelecimento de uma articulação entre a Educação Superior e a saúde, objetivando a formação de profissionais com ênfase na promoção, prevenção, recuperação e reabilitação da saúde, de acordo com os princípios e diretrizes do SUS. Estes movimentos indutores de mudanças, calcados no planejamento estratégico em saúde, passaram a ocupar um espaço importante nos setores saúde e educação, possibilitando o diálogo que permite a construção conjunta de mudanças requeridas pelo quadro epidemiológico e pelas necessidades de reordenação da formação profissional em saúde (Matos, Tomita, 2004).

Assim, com o intuito de realizar a análise situacional das condições de gerenciamento, planejamento e execução de ações de saúde municipais dentro da ESF, foi realizado um projetopiloto pela Faculdade de Odontologia de Araçatuba - Unesp, em um município de pequeno porte da região Noroeste do Estado de São Paulo (Saliba, Moimaz, Garbin, 2007). Foram levantados dados quantitativos e qualitativos a respeito do acesso da população aos serviços de saúde, das características socioeconômicas e culturais dos usuários, do perfil do serviço de saúde local, e um levantamento epidemiológico das condições de saúde bucal da população rural do município. A partir deste estudo foi possível verificar a falta de informações por parte dos usuários sobre o SUS, sobre o direito à saúde e a Estratégia Saúde da Família, bem como a necessidade de conscientização da população sobre a importância do estabelecimento do controle social como uma das estratégias para o fortalecimento do sistema local de saúde. Observou-se também que existiam dificuldades dos municípios em capacitar os recursos humanos para elaborarem ações de saúde, apesar de muitos servidores já terem participado dos Polos de Educação Permanente em Saúde, de responsabilidade do governo estadual. Os resultados 
encontrados destacaram a necessidade de adequação do município aos objetivos e princípios básicos da ESF. Observou-se como a instituição de ensino poderia ser útil ao contribuir para a melhoria da gestão, para a capacitação e atualização dos recursos humanos e gestores da saúde, ao atuar de duas formas: 1) na prestação de serviços aos municípios e 2) na inserção do aluno de graduação e pós-graduação na realidade local por meio de sua participação em projetos na comunidade. Além disso, também o serviço de saúde poderia ser útil à academia, como contrapartida às discussões sobre a formação de recursos humanos, apresentando as qualidades, as deficiências e as necessidades in loco que devem ser levadas em consideração no estabelecimento de uma formação humanística e eficiente, e ao fornecer subsídios para a elaboração de projetos de reestruturação de cursos na área da saúde, como no caso da Odontologia. Assim, é de extrema importância a realização de parcerias entre Universidade e municípios, a fim de fortalecer o sistema de saúde local e também contribuir para a formação acadêmica de profissionais capacitados a atuarem dentro dos princípios do sistema de saúde (Kim, Ross, 2008; Garbin, Saliba, Moimaz, 2006).

O processo ensino-pesquisa-extensão realizado com a imersão dos sujeitos em cenários reais possibilita a integração universidade-comunidade, ampliando a inserção social. Os resultados obtidos com o projeto-piloto permitiram verificar que havia necessidade de se estender o estudo para os municípios adjacentes, na tentativa de subsidiar o planejamento de ações concretas para melhoria das condições de saúde da população da microrregião. Os municípios apresentavam dificuldades para a organização de seus serviços de saúde, seja por falta de recursos financeiros ou recursos humanos tecnicamente capacitados, seja pela participação pouco efetiva da população no processo decisório das ações de saúde e, até mesmo, pela falta de colaboração dessa mesma população às atividades desenvolvidas devido ao desconhecimento sobre o seu sistema de saúde.

Assim, foi proposta a realização de um amplo projeto envolvendo cinco municípios localizados na região noroeste do estado São Paulo, que apresentavam baixo índice de desenvolvimento humano e população estimada entre dois a seis mil habitantes. O objetivo deste trabalho foi apresentar a experiência deste projeto acadêmico como cenário de ensino-pesquisa no SUS.

\section{Métodos}

Este estudo de intervenção foi desenvolvido durante 2003-2006, com a participação de: docentes, alunos de pós-graduação, acadêmicos e técnicos da área da saúde, lideranças das comunidades, prefeitos, secretários municipais de saúde, secretários municipais de educação, membros dos Conselhos Municipais de Saúde e demais servidores do setor saúde dos municípios envolvidos. O projeto foi financiado pela agência de fomento paulista Fapesp.

Foram objetivos deste projeto:

1 Realizar entrevistas para verificar a satisfação da população residente na área urbana como usuária do sistema de saúde, seu conhecimento a respeito da ESF e sua participação no processo decisório das políticas públicas de saúde, com o objetivo de obter dados necessários para a elaboração de diagnóstico situacional (social, ocupacional, econômico e educacional).

2 Realizar um trabalho educativo, domiciliar, com intuito de transferir conhecimentos e conscientizar os membros das famílias moradoras dos municípios participantes a respeito da ESF e da dinâmica de funcionamento do serviço de saúde local, bem como do direito à participação no processo decisório das políticas públicas de saúde (Conselho Municipal de Saúde, Conferência Municipal de Saúde).

3 Capacitar os membros das equipes da ESF para:

- atuarem nas áreas de abrangência das unidades de atendimento;

- construírem uma rede de informação situacional (social, ocupacional, econômica, educacional) destinada a identificar as necessidades reais da população residente nos municípios e sua interação com as condições de saúde bucal.

4 Avaliar os modelos de atenção à saúde implantados nos municípios, de forma que os mesmos atuem no processo de reorganização de seus modelos de atenção. 
5 Capacitar os cirurgiões-dentistas das equipes de saúde para realizarem estudos epidemiológicos das condições de saúde bucal, buscando a construção de um Sistema de Informação em Saúde Bucal da população dos municípios em questão, para planejamento e avaliação das ações do setor saúde.

6 Capacitar os membros dos Conselhos Municipais de Saúde para atuarem de forma mais eficaz e efetiva na construção do sistema municipal de saúde.

Foram contemplados cinco municípios de pequeno porte da Noroeste Paulista. A equipe executora responsável pelo projeto, representada pela universidade, foi dividida em quatro subáreas principais: 1 controle social, por meio do trabalho junto aos Conselhos Municipais de Saúde, 2 avaliação do serviço por meio da ótica do usuário, 3 gestão da saúde, e 4 Estratégia Saúde da Família - permitindo uma melhor organização das atividades e execução da agenda.

Inicialmente, foi feito contato com autoridades locais e com as equipes dos Programas de Saúde por meio de reuniões e visitas aos municípios envolvidos, buscando formalizar as parcerias para execução do projeto.

Foram realizadas diversas visitas técnicas de membros da equipe proponente às equipes da ESF para o acompanhamento da rotina diária do serviço e realização de um relatório preliminar que objetivou conhecer as realidades de cada município. Para a coleta dos dados, análise preliminar da situação e avaliação das mudanças ocorridas após as medidas de intervenção, foram realizadas: pesquisa observacional - utilizando-se diários de campo para anotação da percepção do pesquisador quanto à rotina do serviço - e atividades desenvolvidas; entrevistas semiestruturadas com as equipes da ESF e gestores da saúde para avaliação da organização do serviço, e participação na rotina de atendimento. Também foram realizadas análises documentais, por meio da avaliação crítica dos Planos Municipais de Saúde e dados referentes à gestão.

Uma amostra da população foi entrevistada em domicílio e questionada quanto à sua participação como usuária dos serviços de saúde municipais. Para isso, foi realizado o cálculo da amostra baseado no número total de habitantes do município, considerando uma margem de erro igual a $10 \%$, sendo suficiente para que se fizesse inferência estatística dos dados. Também os conselheiros municipais de saúde foram entrevistados sobre a sua participação e as ações realizadas nos Conselhos Municipais de Saúde.

Os dados foram analisados quantiqualitativamente e apresentados em publicações prévias (Santos et al., 2008; Saliba, 2007). Neste artigo foram descritos os principais resultados encontrados com o foco na integração ensino-pesquisa.

Este projeto foi aprovado pelo Cômite de Ética e Pesquisa em Seres Humanos da Faculdade de Odontologia de Araçatuba - Unesp, seguindo os ditames éticos da Declaração de Helsinque. Foi obtido o Termo de Consentimento Livre e Esclarecido de cada participante.

\section{Resultados e discussão}

A partir das atividades e intervenções executadas (Quadro 1), diversos resultados foram encontrados. A gestão foi dinamizada, o usuário tornou-se mais consciente de seus deveres e direitos, a equipe de saúde foi capacitada e os Conselhos Municipais passaram por reformulações. A integração do município com a Universidade se deu por meio da assessoria da academia na resolução dos nós críticos vivenciados pelos serviços locais de saúde, e pela adequação da formação universitária por meio da contribuição do serviço de saúde. 
Quadro 1. Atividades e intervenções desenvolvidas no projeto. Brasil, 2003-2006.

\begin{tabular}{|l|l|}
\hline Atividades e intervenções & Sujeitos envolvidos \\
\hline $\begin{array}{l}\text { Análise observacional da realização das atividades nas unidades de atendimento } \\
\text { Análise dos Planos Municipais de Saúde e gestão } \\
\text { Readequação dos Planos Municipais de Saúde }\end{array}$ & $\begin{array}{l}\text { Gestores da saúde, equipes de saúde } \\
\text { e usuários }\end{array}$ \\
\hline $\begin{array}{l}\text { Oficinas com as equipes de saúde geral e saúde bucal do PSF para análise das } \\
\text { atividades desenvolvidas nas unidades de atendimento } \\
\text { Elaboração do material didático para capacitação dos membros do PSF } \\
\text { Curso de capacitação dos membros das equipes de saúde geral e saúde bucal do PSF }\end{array}$ & Equipes de saúde \\
\hline $\begin{array}{l}\text { Elaboração do manual para Conselheiros Municipais de Saúde } \\
\text { Curso de capacitação dos Conselheiros Municipais de Saúde }\end{array}$ & Conselhos Municipais de Saúde \\
\hline $\begin{array}{l}\text { Entrevistas com os usuários dos serviços de saúde para verificar o seu grau de } \\
\text { satisfação em relação aos serviços } \\
\text { Elaboração do manual dos usuários do Sistema Único de Saúde. Entrega do manual } \\
\text { em todos os domicílios e realização de trabalho educativo, com a participação dos } \\
\text { agentes comunitários de saúde, após capacitação. Avaliação do conhecimento e } \\
\text { satisfação dos usuários após entrega do manual }\end{array}$ & Usuários de Saúde \\
\hline $\begin{array}{l}\text { Oficinas para avaliação global do projeto: equipe proponente, participantes dos } \\
\text { munićpios, usuários do Sistema de Saúde e gestores } \\
\text { Reunião com os gestores e administradores municipais para avaliação do projeto e } \\
\text { divulgação dos resultados obtidos para a população atendida } \\
\text { Planejamento das ações futuras a serem desenvolvidas pelos serviços de saúde de } \\
\text { cada município }\end{array}$ & Todos \\
\hline
\end{tabular}

A participação dos acadêmicos se deu por meio de estágio de observação e participação nas atividades de campo. Foram discutidos intensivamente os aspectos relacionados à dinâmica do serviço de saúde, princípios e doutrina do SUS, permitindo ao aluno trabalhar os conceitos teóricos na prática. A partir de subprojetos inseridos no projeto principal, iniciações científicas foram desenvolvidas e obtiveram financiamento de agências de fomento.

Este projeto contribuiu com a ampliação dos espaços de intervenção em saúde para a intensificação de canais de integração entre os pares envolvidos - universidade, serviços e comunidade - suportados pelos princípios doutrinários e organizativos do sistema de saúde. A participação da Universidade como incentivadora e promotora dessas ações mostra o papel social da academia como construtora da realidade à qual pertence (Muñoz, Cabieses, 2008).

Seguem abaixo os resultados referentes às quatro subáreas principais:

\section{Gestão da saúde}

Por meio da análise documental, avaliação das atividades de rotina dos serviços e entrevistas com funcionários e gestores, foi verificada a inadequação dos planos municipais de saúde diante dos regulamentos oficiais; problemas no referenciamento dos pacientes para ações de média e alta complexidade, no desenvolvimento de ações intersetoriais, nas estratégias de programação (diagnóstico epidemiológico e avaliação das ações), na infraestrutura e na falta de estabelecimento de objetivos exequíveis aos serviços de saúde. Além disso, muitas unidades de atendimento estavam desorganizadas, sem uma lógica de priorização de atendimento dos pacientes. Muitas delas apresentaram problemas na infraestrutura, como falta de sala de espera e/ou consultório para atendimento ou ambientes pequenos e inapropriados, situação esta observada em outros locais do país (Souza, Roncalli, 2007). Após as oficinas com gestores e equipes de saúde, utilizandose dos mesmos métodos de análise, verificou-se a adequação dos serviços de saúde por meio da reorganização da demanda e a construção e atualização dos mapas territoriais das áreas de abrangência da ESF. 
Uma administração eficaz e interativa dos serviços de saúde, que considere as habilidades individuais de cada servidor, as especificades do local e as expectativas e necessidades dos usuários, leva ao sucesso no planejamento e execução de ações nos serviços de saúde. Diante das transformações ocorridas nos modelos assistenciais em saúde, em nível nacional e internacional, temse discutido a necessidade de se criarem novas definições das responsabilidades dos órgãos gestores federais, estaduais e municipais e da população local, a busca por soluções mais adequadas à resolução dos problemas de acesso e de efetividade da atenção prestada, e de maior impacto sobre os níveis de saúde da população. A divulgação de projetos na área de saúde pública que discutam esses aspectos é de extrema importância, pois permite compartilhar experiências de sucesso e adaptar os métodos utilizados em diferentes ambientes e contextos, possibilitando o avanço epidemiológico e a promoção de ações efetivas em serviços de saúde (Scochi et al., 2008).

\section{Capacitação dos membros da Estratégia Saúde da Família}

Foi realizada uma oficina com todos os membros das equipes da ESF, procedendo-se à análise documental dos relatórios mensais de produtividade e dos planos de trabalho. Esta fase teve como objetivo avaliar o plano de trabalho e verificar a sua rotina e o impacto destas ações sobre os indicadores de saúde da população.

Foi preparado um curso de capacitação dos membros das equipes da ESF com atividades de dispersão e concentração, atividades teóricas e práticas, incluindo: temas relativos à política de saúde, mudança de paradigma no modelo de atenção à saúde, a família como núcleo, organização dos serviços de saúde, humanização do atendimento, sistemas de informação e atenção ao usuário do serviço de saúde. Os membros das equipes da ESF participaram de oficinas para análise crítica do modelo de atenção à saúde vigente em cada município e formulação de novas propostas de ações.

Os membros das equipes de Saúde Bucal, em especial, participaram de um curso teórico para sensibilizá-los sobre a importância do planejamento das ações de saúde, com base em dados da população adscrita, além de serem capacitados para elaborarem estudos epidemiológicos das principais doenças que acometem a cavidade bucal. Foram realizadas reuniões com as equipes para análise e avaliação dos dados e elaboração de um documento oficial apresentado aos gestores e administradores municipais com o intuito de avaliar os resultados encontrados e discutir novas estratégias de ação em saúde bucal, buscando um acompanhamento continuado e permanente das atividades implantadas. Os alunos de pós-graduação, responsáveis pelas atividades ministradas nos cursos juntamente com os docentes, tiveram a oportunidade de aperfeiçoar suas potencialidades como futuros professores.

A ESF constitui-se de equipes multiprofissionais que devem atuar em uma perspectiva interdisciplinar. Estudos têm mostrado como os membros da equipe articulam suas práticas e saberes no enfrentamento de cada situação identificada para proporem soluções conjuntamente e intervir de maneira adequada (Oliveira, Spiri, 2006). A integração entre as equipes deve ser permanentemente estimulada, e a capacitação e atualização constantes permitem a valorização e aperfeiçoamento do grupo.

O contexto da reforma do setor saúde e as transformações vertiginosas na área da saúde tornam a capacitação de recursos humanos em saúde um desafio mediante a reconhecida transitoriedade do saber e das marcantes mudanças no mundo do trabalho em saúde. Em 1996, foi aprovada a Lei de Diretrizes e Bases da Educação Nacional; todavia, sua implementação implica grande desafio na direção de formar profissionais da saúde com competência técnica e política, com compromisso com os princípios da Reforma Sanitária Brasileira e do SUS. As novas diretrizes curriculares na área da saúde explicitam a necessidade de uma metodologia de ensino-aprendizagem que favoreça a integração docente-assistencial, a formação crítica e reflexiva, a responsabilidade compartilhada pelo professor e estudante e a integração entre as instituições de Ensino Superior e os serviços de saúde. A integração docente-assistencial pode ser compreendida como um caminho de aproximação da universidade com os serviços de saúde, sendo esses, nos anos 80 e começo dos anos 90 , restritos aos hospitais de ensino; e a partir da $10^{\circ}$ Conferência Nacional de Saúde revela-se a necessidade de ampliação do campo de ensino englobando todas as unidades do SUS. Mais tarde, em 2005, o PRÓ-SAÚDE retoma essa modalidade, explicitando-a como um vetor importante durante todo o processo de ensino- 
aprendizagem, com integração da orientação teórica com a prática assistencial e com boa articulação entre as atividades teórico-assistenciais em nível individual e coletivo.

Assim, a Constituição Nacional atribuiu ao sistema de saúde a responsabilidade de ordenar a formação profissional na área da saúde. A portaria 198/2004 instituiu a Política Nacional de Educação Permanente em Saúde (PNEPS) como estratégia do SUS para a formação e o desenvolvimento de trabalhadores para o setor da saúde (Brasil, 2004). Neste contexto, a condução locorregional da PNEPS deu-se por meio de um colegiado de gestão configurado como Polo de Educação Permanente em Saúde para o SUS, que funciona como o espaço de articulação de uma ou mais entidades voltado para a formação e educação permanente de recursos humanos em saúde. Essas entidades são vinculadas às universidades ou instituições de Ensino Superior e se integram com as secretarias estaduais e municipais de saúde para implementarem programas de capacitação destinados aos profissionais de Saúde da Família. Os polos de capacitação vêm trabalhando em duplo sentido para o desenvolvimento de conhecimentos, habilidades e atitudes necessárias à nova concepção de trabalho, de forma a preparar os profissionais já formados ou em fase de formação para o enfrentamento de situações e problemas no cotidiano das comunidades. Apesar disso, existem dificuldades que interferem no andamento desses polos, relacionadas com: as deficiências no acompanhamento e avaliação dos processos de capacitação; a inexistência de um banco de dados; a falta de apoio para estudos e pesquisas dos órgãos financiadores, e as falhas na comunicação, entre outras.

Tais instrumentos de poder e de articulação dos quais dispõe o sistema para orientar o processo de formação e a distribuição dos recursos humanos, além de não serem suficientes, não têm sido utilizados na sua totalidade (Almeida, Ferraz, 2008, p. 35). A capacitação dos recursos humanos neste projeto, referente tanto à formação acadêmica do aluno quanto à capacitação e atualização do servidor de saúde, foi realizada com o intuito de colaborar com os polos, agindo como um multiplicador de suas ações, e, ao mesmo tempo, promovendo a integração ensino-serviço.

\section{Capacitação dos membros dos Conselhos Municipais de Saúde}

Foram realizadas atividades práticas destinadas à coleta de dados relativa aos Conselhos Municipais de Saúde. O curso de capacitação para os Conselheiros Municipais de Saúde, com módulos de concentração e dispersão, foi preparado pela equipe e conduzido nos cinco municípios. Como resultado, além da formação e conscientização dos participantes, foram observadas mudanças nas leis do regimento interno dos Conselhos Municipais de Saúde, avaliadas por meio de análise documental, em concordância com a legislação nacional, colocando em prática o controle social.

Muitos membros dos Conselhos não tinham preparo para executar a sua função dentro do comitê. Isso se deve à falta de capacitação e instrução quando aqueles iniciaram a sua participação como conselheiros. O clientelismo e o paternalismo ainda são características marcantes nas relações entre o governo e os grupos de interesse no Brasil, especialmente nas cidades pequenas e nas áreas rurais menos industrializadas do país. Embora os Conselhos de Saúde possam contribuir para a consolidação de formas mais democráticas na representação de interesses, eles têm seu o funcionamento limitado e condicionado pela realidade concreta das instâncias e da cultura política dos municípios brasileiros (Matos, Tomita, 2004). O papel do cidadão que participa dos Conselhos Municipais de Saúde é de representar a sua comunidade e lutar pela preservação e melhoria das condições de saúde e dos serviços ligados a ela. Na perspectiva da gestão participativa, devem ser evitadas dinâmicas dicotômicas entre Sociedade Civil e Estado, promovendo efetivamente espaços dialógicos e de corresponsabilização no processo democrático de construção de políticas públicas de saúde. O envolvimento de representantes dos movimentos sociais organizados não deve estar restrito à apresentação de demandas, sendo fundamental a criação de espaços acolhedores em que as lideranças sociais encontrem um campo em que seja possível propor alternativas de intervenção, e não apenas demandar do governo respostas ou soluções diante dos problemas existentes (Costa, Lionço, 2006). O esforço do projeto em capacitar os conselheiros e também usuários de saúde foi para auxiliar na garantia do controle social de forma efetiva em âmbito municipal, o que gera resultados positivos em um nível estadual e federal. 
As práticas e mecanismos de participação social no campo da saúde constituem referências para a democracia participativa no Brasil. A universalidade do direito à saúde, na perspectiva da integralidade da atenção e do cuidado e da participação social na gestão das políticas de saúde, conforma um patrimônio da sociedade em seu processo de democratização (Cortes, 2002, p. 19). Considerando que a participação social e a democracia participativa atuam na qualificação da gestão publica, é de grande relevância a organização e atuação dos Conselhos Municipais de Saúde.

\section{Avaliação do serviço pelos usuários}

Por meio de oficinas, foram capacitados os profissionais das equipes da ESF a fim de que estes elaborassem os instrumentos necessários para verificar a satisfação dos usuários do Sistema Municipal de Saúde. Os profissionais foram capacitados para preparar a coleta dos dados, definir o número amostral e as estratégias de abordagem dos indivíduos. Foram realizadas entrevistas domiciliares em todos os municípios com a participação das equipes de saúde e proponentes do projeto.

Com base nos resultados obtidos, foi possível constatar a falta de conhecimento dos usuários sobre o serviço de saúde que utilizam, além do desinterese ou ignorância quanto aos seus direitos e deveres. Quanto maior o nível de informação do usuário sobre objetivos, atividades e regras de funcionamento da ESF, maior o grau de satisfação em relação à estratégia. O nível de informação está condicionado pelo grau de escolaridade do usuário e a eficácia das estratégias de comunicação e informação em saúde utilizada pela equipe (Trad et al., 2002). Isto fica bem evidente ao se constatar o baixo nível socioeconômico e educacional da população avaliada.

Assim, foi determinado o estabelecimento de ações que permitissem aos usuários a conscientização de seu papel como participante ativo na construção de seus serviços de saúde. Foram realizadas reuniões com o intuito de elaborar um manual com informações básicas sobre o Sistema Único de Saúde direcionadas aos usuários. A partir destas, foi confeccionado o Manual do Usuário, distribuído para todos os habitantes dos municípios envolvidos.

A medida de satisfação do usuário é uma avaliação pessoal dos cuidados e serviços de saúde que são dispensados. Isso implica um julgamento sobre a qualidade dos serviços ofertados como medida de otimização das ações (Ware et al., 1983). A perspectiva do usuário fornece informação essencial para se completar e equilibrar a qualidade dos serviços (Emmi, Barroso, 2008; van Stralen et al., 2008). Alguns autores consideram que todas as pesquisas no âmbito da satisfação do usuário devem propiciar aperfeiçoamento para o cotidiano dos serviços de saúde e avanços significativos para a gestão dos mesmos (Esperidião, Trad, 2005).

Ao final de cada etapa do projeto, foram aplicadas avaliações qualitativas com o intuito de medir o grau de aprendizado e corrigir eventuais barreiras na geração e transmissão de conhecimentos.

No final, fez-se uma análise global do projeto, avaliando-se a equipe proponente, os usuários do sistema de saúde, os gestores e os diversos segmentos do setor saúde que participaram das atividades e ações desenvolvidas.

O projeto descrito neste trabalho permitiu auxiliar os serviços de saúde na organização de suas atividades, na adequação às diretrizes do sistema de saúde nacional e na implementação correta da Estratégia Saúde da Família. A atuação participativa dos acadêmicos e alunos de pós-graduação, profissionais de saúde, conselheiros e usuários demonstrou a eficiência e o alcance dos objetivos do projeto. Os serviços de saúde colaboraram de forma importante na discussão sobre a formação de recursos humanos, indicando deficiências na formação universitária e permitindo que mudanças e adaptações nos conteúdos curriculares fossem realizadas. Foi uma importante ferramenta para os municípios e a Universidade, na medida em que capacitou e deu suporte aos gestores e demais servidores, e promoveu aprendizagem aos acadêmicos e pós-graduandos. A academia cumpriu seu papel social de prestação de serviço e apoio à comunidade, de modo direto e indireto.

De acordo com as Novas Diretrizes Curriculares Nacionais dos cursos de graduação em Odontologia (Brasil, 2002), o cirurgião-dentista necessita ser sujeito de um processo de qualificação profissional, visando integrar uma equipe e desenvolver ações programáticas inseridas nesta estratégia 
de intervenção populacional baseada no território com vistas à efetivação do SUS (Matos, Tomita, 2004, p. 1538). A inserção do aluno no processo de desenvolvimento e execução do projeto permitiu a vivência da realidade do serviço como sujeito ativo, de forma que fossem estimulados o senso crítico, habilidades cognitivas, capacidade de resolução e adaptação. Em todas as vertentes do projeto, o acadêmico e pós-graduando foram atuantes, participando desde o planejamento, trabalho de campo até a análise dos resultados. A vivência destas atividades serviu como laboratório de aprendizagem, permitindo aos alunos um conhecimento mais fidedigno, e mais consciente, das ações de políticas públicas. O projeto proporcionou o estreitamento do vínculo dos acadêmicos e pós-graduandos com os docentes, os serviços de saúde e usuários do SUS, assim como contribuiu para uma formação profissional mais humanitária.

A literatura apresenta diversos estudos voltados apara a administração de serviços públicos realizada de forma inteligente e direcionada às necessidades locais. Na Bolívia, foram alcançados resultados satisfatórios com a implementação de projetos destinados à melhoria da qualidade dos serviços de saúde, por meio da capacidade de resolução das medidas propostas; da avaliação de problemas relacionados à administração dos serviços de saúde; do uso de técnicas simples e modernas adaptadas ao conhecimento local, e do treinamento de recursos humanos durante a execução do projeto (Ferrelli et al., 1997). Ao comparar os projetos, fica fácil perceber a similaridade de situações e de resultados. De uma maneira ampla, as dificuldades e carências entre os países, em especial na América Latina, são as mesmas, havendo a necessidade de se discutirem os problemas para que propostas sejam compartilhadas de forma a se encontrarem soluções inteligentes, eficazes e de baixo custo.

Todo trabalho de campo não é fácil de ser conduzido. É necessário planejar antecipadamente, de um modo seguro e realista, as atividades a serem realizadas. Mesmo com um rigoroso plano a priori, dificuldades serão encontradas, havendo, muitas vezes, a necessidade de mudanças e alterações dos planos estabelecidos. Tal fato aconteceu diversas vezes durante a execução deste projeto, e para isso foi necessário o controle dos objetivos definidos para a intervenção, e a avaliação permanente dos resultados obtidos. Também o auxílio financeiro é necessário, pois os custos são elevados. Alguns pontos foram extremamente importantes para o desenvolvimento das ações: o estabelecimento de uma agenda, com atualização constante das atividades a serem realizadas; controle do material utilizado em campo, como as fichas de entrevistas; e organização e hierarquização da equipe, com distribuição de tarefas para subgrupos de participantes. Como fatores críticos que contribuíram para o sucesso da iniciativa, podem ser citados: a parceria da instituição de ensino com as prefeituras, por meio da contrapartida destas nas atividades desenvolvidas, como, por exemplo, a disponibilização de transporte e alimentação para a equipe. Do mesmo modo, a recepção dos pesquisadores pelos usuários foi extremamente positiva. Como principal dificuldade destacou-se a falta de cooperação de um gestor e o transporte entre os municípios. Embora as distâncias não fossem tão grandes, foi difícil conseguir os meios de transporte, apesar dos esforços da instituição de ensino e prefeituras que, na medida do possível, cederam os seus carros para a equipe.

\section{Conclusão}

Essa experiência serviu como laboratório de aprendizagem e de pesquisa, fazendo-se ciência a partir da vivência in loco da realidade do SUS e contribuindo para uma formação profissional mais humanitária baseada em cenários reais. O projeto também alcançou resultados positivos ao favorecer a melhoria da gestão em saúde, por meio de ações conjuntas entre Universidade e serviços públicos de saúde. Diante da atual conjectura nacional, o projeto ganhou destaque por contribuir com o fortalecimento e consolidação das políticas públicas de saúde. 


\section{Colaboradores}

Os autores trabalharam juntos em todas as etapas de produção do manuscrito.

\section{Referências}

ALMEIDA, L.P.G.; FERRAZ, C.A. Políticas de formação de recursos humanos em saúde e enfermagem. Rev. Bras. Enferm., v.61, n.1, p.31-5, 2008.

BRASIL. Conselho Nacional de Educação. Diretrizes Curriculares Nacionais do curso de graduação em Odontologia. Resolução CNE/CES 3/2002. Diário Oficial da União, Brasília, 4 março 2002. Seção 1, p.10.

. Ministério da Saúde, Conselho Nacional de Saúde. Resolução 333/03. Coletânea de Normas para o Controle Social no SUS. Brasília: Ministério da Saúde, 2006. seção I. Ministério da Saúde. Portaria n.198. Diário Oficial da União, Brasília, n.32/2004, Ministério da Saúde. Lei 8080/90. Brasília: Ministério da Saúde, 1990a. . Secretaria da Saúde. Lei 8142/90. Brasília: Secretaria da Saúde, 1990b.

CORTES, S.M.V. Construindo a possibilidade de participação dos usuários: conselhos e conferências no Sistema Único de Saúde. Sociologias, n.7, p.18-49, 2002.

COSTA, A.M.; LIONÇO, T. Democracia e gestão participativa: uma estratégia para a eqüidade em saúde? Saude Soc., v.15, n.2, p.47-55, 2006.

EMMI, D.P.; BARROSO, R.F.F. Avaliação das ações de saúde bucal no Programa Saúde da Família no distrito de Mosqueiro, Pará. Cienc. Saude Colet., v.13, n.1, p.35-41, 2008.

ESCOREL, S. et al. The Family Health Program and the construction of a new model for primary care in Brazil. Rev. Panam. Salud Publica, v.21, n.2-3, p.164-76, 2007.

ESPERIDIÃO, M.; TRAD, L.A.B. Avaliação da satisfação de usuários. Cienc. Saude Colet. v.10, supl, p.303-12, 2005.

FERRELLI, R. et al. Strengthening local health care management in Bolivian districts through participatory operational research. Int. J. Health Plann. Manage., v.12, n.1, p.29-50, 1997.

GARBIN, C.A.S.; SALIBA, N.A.; MOIMAZ, S.A.S. O papel das Universidades na formação de profissionais na área de saúde. Rev. ABENO, v.6, n.1, p.6-10, 2006.

GONZÁLEZ, G.G. Health sector reform and management models. Rev. Panam. Salud Publica, v.9, n.6, p.406-12, 2001.

KIM, Y.D.; ROSS, L. Developing service user involvement in the South Korean disability services: lessons from the experience of community care policy and practice in UK. Health Soc. Care Commmunity, v.16, n.2, p.188-96, 2008

MATOS, P.E.S.; TOMITA, N.E. A inserção da saúde bucal no Programa Saúde da Família: da universidade aos pólos de capacitação. Cad. Saude Publica, v.20, n.6, p.1538-44, 2004.

MUÑOZ, M.; CABIESES, B. Universities and health promotion: how can the two come together? Rev. Panam. Salud Publica, v.24, n.2, p.139-46, 2008.

OLIVEIRA, E.M.; SPIRI, W.C. Programa Saúde da Família: a experiência de equipe multiprofissional. Rev. Saude Publica, v.40, n.4, p.727-33, 2006.

SALIBA, N.A. Projeto promoveu intercâmbio entre gestores de saúde usuários do SUS. Sp. Gov., v.9, p.34-37, 2007. 
SALIBA, N.A.; MOIMAZ, S.A.S.; GARBIN, C.A.S. Parceria pela saúde - projeto desenvolvido no interior do estado de São Paulo, financiado pela Fapesp, integra instituições educacionais da área da saúde, governo e sociedade civil. Sp. Gov., v.4, p.3437, 2007.

SANTOS, K.T. et al. Agente comunitário de saúde: perfil adequado a realidade do Programa Saúde da Família?. Cienc. Saude Colet. (online), 2008. Disponível em: $<$ http://www.abrasco.org.br/cienciaesaudecoletiva/artigos/artigo_int.php?id_ artigo $=2104>$. Acesso em: 2 set. 2009.

SCOCHI, M.J. et al. Capacitação das equipes locais como estratégia para a institucionalização da avaliação em saúde. Cad. Saude Publica, v.24, supl.1, p.S183-92, 2008.

SOUZA, T.M.S.; RONCALLI, A.G. Saúde Bucal no Programa Saúde da Família: uma avaliação do modelo assistencial. Cad. Saude Publica, v.23, n.11, p.2727-39, 2007.

TRAD, L.A.B. et al. Estudo etnográfico da satisfação do usuário do Programa de Saúde da Família (PSF) na Bahia. Cienc. Saude Colet., v.7, n.3, p.581-9, 2002.

VAN STRALEN, C.J. et al. Percepção dos usuários e profissionais de saúde sobre atenção básica: comparação entre unidades com e sem saúde da família na Região Centro-Oeste do Brasil. Cad. Saude Publica, v.24, supl.1, p.S148-58, 2008.

WARE, J.E. et al. Defining and measuring patient satisfaction with medical care. Evaluation Program Planning, v.6, n.3-4, p.247-63, 1983.

MOIMAZ, S.A.S. et al. Prácticas en la enseñanza-aprendizaje con base en escenarios reales. Interface - Comunic., Saude, Educ., v.14, n.32, p.69-79, jan./mar. 2010.

El proceso enseñanza-investigación-extensión realizado con la inmersión de los sujetos en escenarios reales posibilita la integración universidad-comunidad ampliando la inserción social. El objetivo de este trabajo ha sido de presentar la experiencia de un proyecto conducido en cinco municipios brasileños con la participación de docentes, pos-graduandos y académicos con la cooperación de alcaldías y trabajadores de salud como escenario de enseñanza-investigación del Sistema Único de Salud. Se han realizado talleres y cursos de capacitación con los equipos de salud, gestores y consejeros de salud, visitas técnicas a los municipios para evaluación de situación y supervisión de actividades, evaluación de la satisfacción de los usuarios y formación de jefaturas populares. Se observa mejoría en la organización de los servicios y estímulo al fortalecimiento del vínculo entre los usuarios y los prestadores de servicios. Esta experiencia ha servido como laboratorio para aprendizaje y investigación, haciéndose ciencia a partir de la vivencia in loco de la realidad del Sistema Único de Salud y contribuyendo para una formación profesional más humanitaria basada en escenarios reales.

Palabras clave: Sistema Único de Salud. Enseñanza. Academias e Institutos. Recursos humanos en salud. Investigación sobre servicios de salud. 